\title{
Durée de vie d'un carbure P20 pour le chariotage d'un acier C38 à rugosité contrôlée
}

\author{
Ali Benamar ${ }^{1, a}$, Geneviève Inglebert² ${ }^{2}$ Mo Mohamed Boumahrat ${ }^{2}$ \\ 1 Département de Mécanique, ENSET Oran, BP 1523, 31000 Oran, Algérie \\ 2 LISMMA, EA2336, groupe tribologie, ISMEP, 3 rue Fernand Hainaut, 93407 St Ouen, France
}

Reçu le 20 juin 2003, accepté le 2 juillet 2004

\begin{abstract}
Résumé - Ce travail a pour objectif de déterminer la durée de vie d'un outil en carbure métallique P20 pour une opération de chariotage en tournage, en vue de l'obtention d'une rugosité imposée. L'amélioration des essais permet d'apprécier les performances optimales d'usinage et d'assurer la rugosité imposée par le dessin de définition d'une pièce mécanique par une étude expérimentale. Les principaux facteurs influençant la rugosité ont été recensés systématiquement en vue d'assurer les conditions optimales d'usinage. La variation de la rugosité en fonction de la durée de vie de l'outil, ainsi que sa fiabilité, a été étudiée. Ce travail est complété par l'élaboration d'un modèle stochastique traduisant l'évolution de la rugosité afin d'exprimer les limites d'utilisation de l'outil pour le chariotage de manière préventive. Le modèle permet de déterminer le temps de réglage et de le considérer comme contrainte pour le respect de la rugosité lors d'un travail de série en usinage conventionnel.
\end{abstract}

Mots clés : Tribologie / intégrité de surface / rugosité / usinage / réglage de machine-outil / usure

Abstract - Life of a P20 carbide used in a lathing process on a C38 steel with controlled roughness. This work aims at life evaluation of a P20 carbide used in lathing process on a C38 steel with controlled roughness; the optimal performances with respect of the roughness imposed by drawing or the part have been achieved through improved experimental tests. The main parameters which affect the roughness were systematically investigated, to insure the optimal conditions. The roughness variation and tool reliability had been studied versus tool life. This work is completed by a stochastic model; describing the roughness evolution and predicting the limits for using the lathing tool. The fixing time is defined by this model.

Key words: Tribology / surface integrity / roughness / manufacturing / fixing time / wear

\section{Importance de l'intégrité de la qualité des surfaces usinées}

La qualité de l'état d'une surface usinée a une très grande importance pour les fonctions de résistance au frottement, au roulement, à la fatigue, à la corrosion, au matage, à l'écoulement des fluides, à l'étanchéité, l'adhésivité, etc. La recherche de la meilleure qualité fonctionnelle au moindre coût et la surveillance de la qualité en fabrication mécanique pour assurer l'interchangeabilité, nécessitent préalablement un moyen précis de mesure industriel des irrégularités et défauts microgéométriques de surfaces par le contrôle de la rugosité $[1,2]$.

En service, l'usure des organes touche en premier lieu les couches superficielles du métal, d'où l'importance du choix de la qualité d'usinage et de son intégrité à l'égard

\footnotetext{
a Auteur correspondant : benamar_dz@yahoo.fr
}

du fonctionnement et de la longévité d'un ensemble [3]. L'intégrité de la qualité des surfaces des pièces élaborées et celles des outils coupants susceptibles de répondre aux besoins de la technologie moderne (rendement et productivité élevés tout en respectant la rugosité spécifiée par le dessin de définition) ont un rôle de premier ordre en construction mécanique [4]. C'est pourquoi la connaissance approfondie des relations exprimant l'influence des facteurs de base de l'usinage par enlèvement de matière (propriétés du matériau usiné, vitesse de coupe, avance, profondeur de passe, géométrie de la partie active de l'outil, lubrifiant, etc.) sur l'état de surface travaillé $[5,6]$ est importante. Aussi l'élaboration d'une méthodologie de corrélation entre la rugosité et la limite d'utilisation de l'outil coupant lors du processus d'usinage en tournage est essentielle pour un travail de série; c'est l'objet de cette étude. 


\section{Nomenclature}

\begin{tabular}{|c|c|c|}
\hline$R$ & rugosité & $(\mu \mathrm{m})$ \\
\hline$R_{\mathrm{g}}$ & rugosité théorique de génération & \\
\hline$R_{\mathrm{a}}$ & rugosité moyenne arithmétique & \\
\hline$R_{\mathrm{z}}$ & rugosité maximale & \\
\hline$R_{\mathrm{t}}$ & rugosité totale & \\
\hline$R_{\mathrm{M}}$ & valeur maximale de la rugosité imposée & \\
\hline$R_{\mathrm{m}}$ & valeur minimale de la rugosité imposée & \\
\hline$\delta R$ & variable aléatoire & \\
\hline$\sigma$ & écart type & \\
\hline$\mu$ & moyenne arithmétique statistique & \\
\hline$t$ & variable de la répartition STUDENT & \\
\hline$\tau$ & temps de coupe & $(\min )$ \\
\hline$c$ et $m$ & constantes qui dépendent de la coupe & \\
\hline$V_{\mathrm{c}}$ & vitesse de coupe & $\left(\mathrm{m} \cdot \min ^{-1}\right)$ \\
\hline$s$ & avance & $\left(\mathrm{mm} \cdot \mathrm{tr}^{-1}\right)$ \\
\hline$f$ & profondeur de passe & $(\mathrm{mm})$ \\
\hline$\alpha$ & angle de dépouille & $\left({ }^{\circ}\right)$ \\
\hline$\gamma$ & angle d'attaque & $\left({ }^{\circ}\right)$ \\
\hline$r_{\varepsilon}$ & rayon d'arrondi du bec de l'outil & $(\mathrm{mm})$ \\
\hline$V_{\mathrm{b}}$ & usure frontale de l'outil & $(\mathrm{mm})$ \\
\hline$V_{\mathrm{r}}$ & usure radiale de l'outil & $(\mathrm{mm})$ \\
\hline$K_{\mathrm{t}}$ & usure en cratère de l'outil & $(\mathrm{mm})$ \\
\hline P20 & nuance de carbure de l'outil & \\
\hline C38 & acier XC38f & \\
\hline
\end{tabular}

\section{Modèle mathématique de la rugosité des surfaces usinées par enlèvement de matière}

\subsection{Introduction}

Si les usinages des pièces étaient obtenus avec des systèmes technologiques idéaux (dans lesquels il n'existe pas d'erreurs d'usinage), la rugosité des surfaces serait déterminée par les paramètres géométriques de l'outil et les paramètres cinématiques du processus de génération. Cette hypothèse appliquée au profil de la pièce usinée met en évidence la rugosité théorique de génération $\left(R_{\mathrm{g}}\right)$. Mais les usinages ont lieu dans les systèmes technologiques réels, qui font inévitablement intervenir des erreurs d'usinage, et la rugosité obtenue diffère de la rugosité théorique $[6,7]$.

\subsection{Caractère réel de la rugosité obtenue}

On peut considérer que la rugosité $R$ des surfaces usinées par enlèvement de matière $\left(R_{\mathrm{a}}, R_{\mathrm{z}}, R_{\mathrm{t}}\right)$ a un caractère stochastique, sa loi de variation présente deux composantes :

- Une composante déterministe : $R_{0}=R_{\mathrm{g}}+\Delta R$.
- Une composante aléatoire $: \delta R$

$$
R=R_{0}+\delta R
$$

La composante déterministe $R_{0}$ de la fonction $R$ est connue ou déterminée par l'expérimentation. Elle est le résultat de l'influence des facteurs systématiques qui se manifestent pendant le processus d'usinage.

\subsection{Valeurs extrêmes de la fonction}

La valeur maximale $\left(R_{\mathrm{M}}\right)$ et respectivement la valeur minimale $\left(R_{\mathrm{m}}\right)$ sont a priori imposées comme limite de rugosité sur le dessin de définition de la pièce.

La variable aléatoire $\delta R$, engendrée par des erreurs aléatoires d'usinage, présente une répartition d'après la loi normale (Laplace Gauss). Cette répartition est centrée, de moyenne arithmétique nulle $(\mu=0)$ et d'écart type $\sigma$.

$\delta R$ a un intervalle de confiance $[-t \sigma ;+t \sigma]$ pour une probabilité $(P)$, c'est-à-dire :

$$
-t \sigma \leq \delta R \leq+t \sigma
$$

où $t$ est la variable de la répartition de STUDENT.

L'intervalle de confiance de la fonction $R$, pour un temps de coupe $\tau$ (Fig. 1) est :

$$
\left[R_{\mathrm{g}}+\Delta R-t \sigma ; R_{\mathrm{g}}+\Delta R+t \sigma\right]
$$




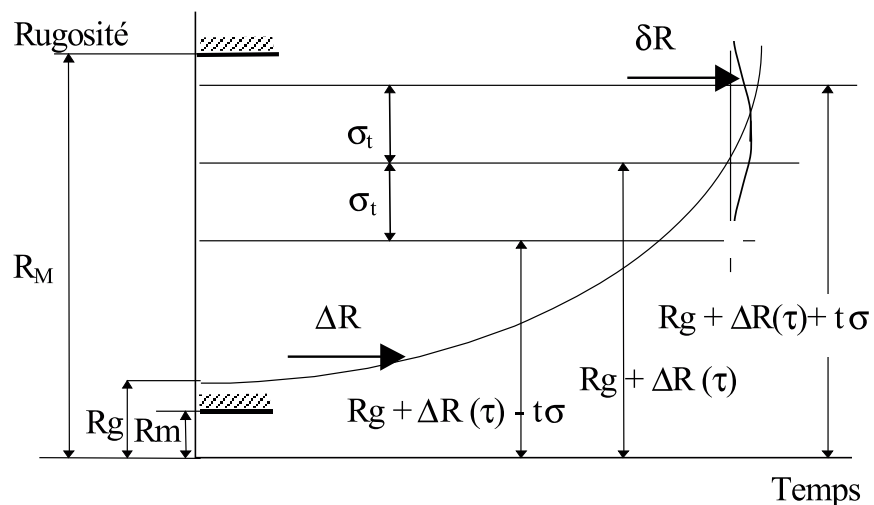

Fig. 1. Variation de la rugosité.

où

$$
R_{\mathrm{g}}+\Delta R(\tau)-t \sigma \leq R(\tau) \leq R_{\mathrm{g}}+\Delta R(\tau)+t \sigma
$$

D'après (1) on peut écrire :

$$
R_{0}(\tau)-t \sigma \leq R(\tau) \leq R_{0}(\tau)+t \sigma
$$

La relation précédente doit être satisfaite pour les valeurs extrêmes de la fonction $R$ (Fig. 1). Ainsi on a les restrictions générales suivantes :

$$
R_{\mathrm{m}} \leq R_{0}(\tau)-t \sigma \leq R(\tau) \leq R_{0}(\tau)+t \sigma \leq R_{\mathrm{M}}
$$

La fonction $R_{0}$ est en général une fonction du temps ayant dans un cycle d'usinage comme valeurs extrémales minimales $\left(R_{0 \mathrm{~m}}\right)$ et maximales $\left(R_{0 \mathrm{M}}\right)$. Pour ne pas dépasser les limites de rugosité imposées, les restrictions (5) doivent être satisfaites aussi pour les valeurs extrémales de la fonction $R_{0}$ :

$$
R_{\mathrm{m}} \leq R_{0 \mathrm{~m}}-t \sigma \leq R \leq R_{0 \mathrm{M}}+t \sigma \leq R_{\mathrm{M}}
$$

À la limite les inégalités (6) deviennent :

$$
R_{0 \mathrm{~m}}=R_{\mathrm{m}}+t \sigma \quad \text { et } \quad R_{0 \mathrm{M}}=R_{\mathrm{M}}-t \sigma
$$

Les dernières relations précisent les valeurs extrémales de la fonction $R_{0}$ de manière à ne pas dépasser les limites de rugosités imposées (Fig. 1).

La fonction $R_{0}$ dépend des paramètres du régime de coupe et des paramètres géométriques de l'outil coupant; ainsi, une des relations (7) ou les deux peuvent être utilisées pour établir les conditions de coupe assurant la rugosité imposée.

Si par exemple la fonction $R_{0}$ est toujours croissante en fonction du temps d'usinage dans l'intervalle $\left[R_{0 \mathrm{~m}} ; R_{0 \mathrm{M}}\right]$; on peut utiliser la première relation de $(7)$ pour déterminer les conditions de coupe; la deuxième relation mise sous la forme (8), peut ensuite servir pour déterminer la durée de vie de l'outil en vue de respecter la rugosité imposée

$$
R_{0}(\tau)=R_{\mathrm{M}}-t \sigma
$$

\section{Méthodologie de recherche théorique}

Les recherches concernant la rugosité ont mis en évidence que la loi de variation de la rugosité en fonction du temps d'usinage est de type polytropique [8] ; cette loi peut être exprimée par la relation suivante :

$$
R=c \tau^{m}
$$

$\tau$ : représente le temps de coupe; $c$ et $m$ sont des constantes qui dépendent des conditions de coupe utilisées lors de l'usinage.

Dans un système d'axe en coordonnées logarithmiques, la relation (9) représente l'équation d'une droite dont $m$ est la pente. L'ensemble des résultats est traité par la technique classique des moindres carrés.

\section{Méthodologie de recherche expérimentale}

\subsection{Plan d'expériences}

Les résultats des essais sur la rugosité présentent une dispersion importante. C'est pourquoi il était nécessaire d'effectuer un minimum de cinq essais dans des conditions identiques pour obtenir une valeur moyenne significative et de mettre en œuvre un plan d'expériences afin d'optimiser le nombre d'essais. Ces expériences permettent la détermination de l'écart type $\sigma$ ainsi que des valeurs affinées de $c$ et $m$. En utilisant les techniques de sondage statistique en petites quantités, nous avons développé un plan d'expériences sur 5 lots de 10 pièces, le temps d'usinage étant considéré comme variable [9]. Les autres paramètres influençant le processus de coupe, tels que la vitesse de coupe, l'avance, la profondeur de passe, l'arrondi du bec de l'outil, la nuance et la géométrie de l'outil sont fixés selon l'étude préliminaire présentée ci-après et les résultats expérimentaux des références [10,11]. Les essais relatifs à cette étude ont été effectués en chariotage sur des pièces cylindriques en acier C38 de diamètre $100 \mathrm{~mm}$ et de longueur $150 \mathrm{~mm}$ au département de mécanique de l'ENSET d'Oran; l'outil était un carbure métallique P20 [12].

\subsection{Présentation des résultats}

\subsection{1 Étude préliminaire aux essais}

Puisque les paramètres de coupe influencent la rugosité, il était utile de connaître plus en détail l'évolution de la rugosité en fonction de la vitesse de coupe, de l'avance et de l'usure. Cela a fait l'objet d'une étude préliminaire dont les résultats ont permis d'optimiser la rugosité recherchée.

Les essais étudiant l'effet de la vitesse de coupe sur la rugosité $\left(R_{\mathrm{a}}=f\left(V_{\mathrm{c}}\right)\right)$, nous ont donné les valeurs de la vitesse de coupe optimale pour ce matériau qui sont comprises entre 220 et $280 \mathrm{~m} \cdot \mathrm{min}^{-1}$ (Fig. 2), sous les conditions de coupe données par le tableau 1. 
Tableau 1. Valeurs des paramètres de coupe et géométrie de l'outil.

\begin{tabular}{ccccccc}
\hline$V_{\mathrm{c}}\left(\mathrm{m} \cdot \mathrm{min}^{-1}\right)$ & $s\left(\mathrm{~mm} \cdot \mathrm{tr}^{-1}\right)$ & $f(\mathrm{~mm})$ & $\alpha\left(^{\circ}\right)$ & $\gamma\left(^{\circ}\right)$ & $r_{\varepsilon}(\mathrm{mm})$ & outil \\
\hline $220-280$ & 0,1 & 0,5 & 6 & 8 & 0,5 & $\mathrm{P} 20$ \\
\hline
\end{tabular}

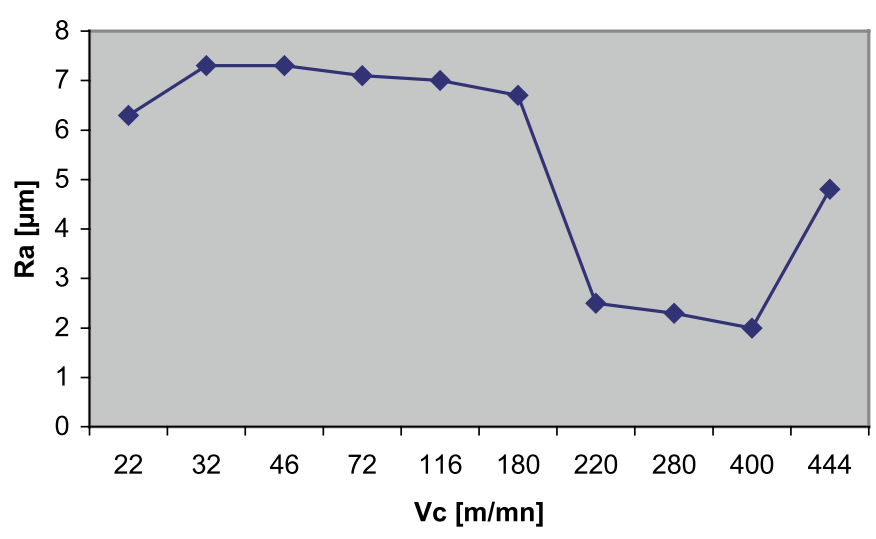

Fig. 2. Influence de la vitesse de coupe sur la rugosité. Outil P20;s=0,1 mm.tr ${ }^{-1} ; f=0,5 \mathrm{~mm} ; \alpha=6^{\circ} ; \gamma=8^{\circ}$; $r_{\varepsilon}=0,5 \mathrm{~mm}$.

Nous remarquons que la valeur de vitesse de coupe de l'ordre de $400 \mathrm{~m} \cdot \mathrm{min}^{-1}$ permet d'obtenir une rugosité légèrement inférieure mais cette dernière n'a pas été prise en considération du fait de l'usure importante engendrée par cette vitesse [4].

- L'aspect d'état de surface (rugosité) est lié aux déplacements successifs du bec de l'outil ainsi qu'au processus d'interaction géométrique entre l'outil et la pièce. Le rayon du bec de l'outil $\left(r_{\varepsilon}\right)$ (Fig. 2) à une influence sur la rugosité [4], la valeur de ce paramètre est fixée à $r_{\varepsilon}=0,5 \mathrm{~mm}$.

- La rugosité dépend aussi de manière significative de l'avance $(s)$; nous avons retenu une valeur optimale de $: s=0,1 \mathrm{~mm} \cdot \mathrm{tr}^{-1}$ (Fig. 3), sous les conditions d'usinage citées ci-dessus avec une vitesse de coupe : $V_{\mathrm{c}}=$ $251 \mathrm{~m} . \mathrm{min}^{-1}$. Cette valeur est relative à la vitesse de rotation disponible sur la machine utilisée (tour Cholet), qui est de 1600 tr. $\mathrm{min}^{-1}$ et permet de rester dans la gamme de vitesses de coupe citée ci-dessus comprise entre 220 et 280 m.min ${ }^{-1}$ (Fig. 2). Étant donné que notre étude porte sur le chariotage de finition, la profondeur de passe $(f)$, ne doit pas être importante mais doit rester néanmoins supérieure à la valeur du copeau minimum d'usinage en tournage; elle est fixée à $f=0,5 \mathrm{~mm}[10,13]$.

- Généralement, lors de l'usinage deux types d'usure se manifestent : usure frontale $\left(V_{\mathrm{b}}\right)$ et usure radiale $\left(V_{\mathrm{r}}\right)$ (Fig. 4) [14]. C'est l'usure frontale qui affecte le plus la rugosité [15]. Les essais effectués ont permis de préciser l'évolution de la rugosité en fonction de l'usure frontale (Fig. 5). D'après cette figure, nous remarquons une augmentation sensible de l'usure pour la première période, ce qui est conforme à l'allure classique de l'usure des matériaux dans la période de rodage. Cette rugosité tend à se stabiliser sur le deuxième palier à partir de $V_{\mathrm{b}}=$ 0,22 $\mathrm{mm}$, ce qui donne un critère définissant le début

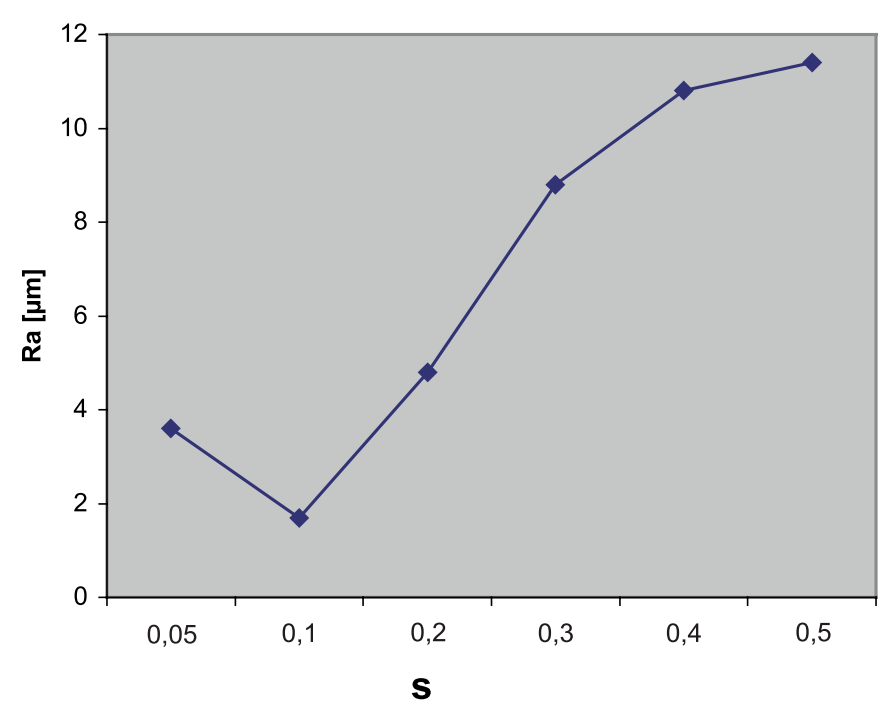

Fig. 3. Influence de l'avance sur la rugosité. Outil P20; $V_{\mathrm{c}}=$ $251 \mathrm{~m} \cdot \min ^{-1} ; f=0,5 \mathrm{~mm} ; \alpha=6^{\circ} ; \gamma=8^{\circ} ; r_{\varepsilon}=0,5 \mathrm{~mm}$.

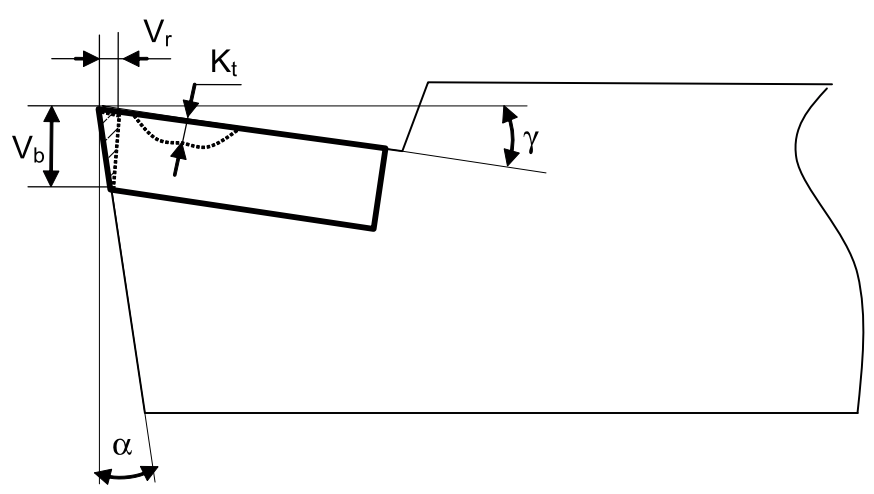

Fig. 4. Géométrie de l'outil.

d'utilisation effective de l'outil pour une bonne reproductibilité [16]. La valeur limite de cette usure est de l'ordre de $1 \mathrm{~mm}$ selon la figure 5 .

\subsubsection{Essais propres}

Avec le réglage des différents paramètres déjà choisis : vitesse de coupe $\left(V_{\mathrm{c}}\right)$, avance $(s)$, profondeur de passe $(f)$, valeur limite d'usure frontale $\left(V_{\mathrm{b}}\right)$, géométrie de l'outil $(\alpha, \gamma)$, rayon du bec de l'outil $\left(r_{\varepsilon}\right)$, les essais sur l'évolution de la rugosité en fonction du temps de coupe $\left(R_{\mathrm{a}}=f(\tau)\right)$ ont été effectués; ils permettent de se prononcer sur la durée de vie de l'outil P20 utilisé en fonction du critère de rugosité retenu. Cette rugosité a été mesurée à l'aide d'un rugosimètre électronique à palpeur inductif (Hommel Werke), avec une longueur de base de $2 \mathrm{~mm}$ et un cut-off de 0,75 $\mathrm{mm}$, au Laboratoire 


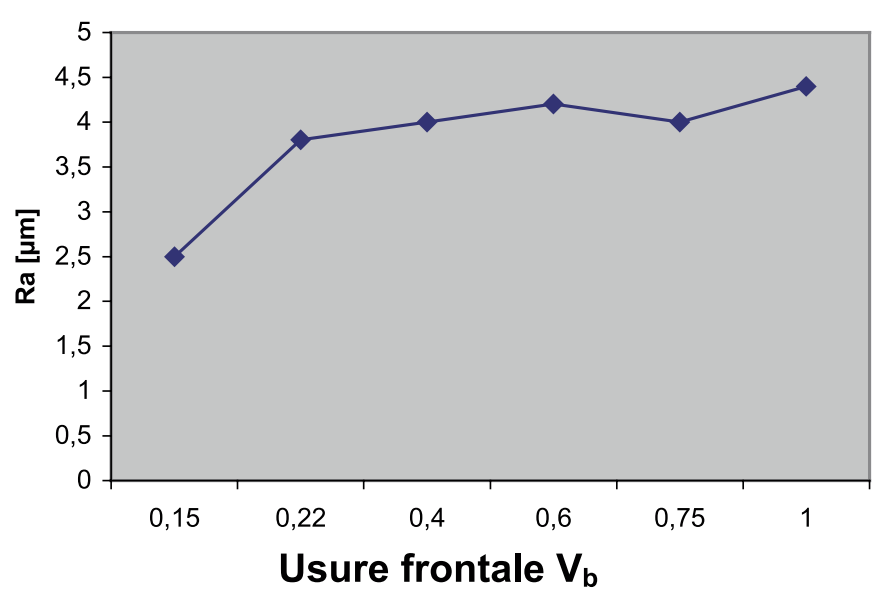

Fig. 5. Influence de l'usure sur la rugosité. Outil P20; $V_{\mathrm{c}}=$ $251 \mathrm{~m} \cdot \mathrm{min}^{-1} ; s=0,1 \mathrm{~mm} \cdot \mathrm{tr}^{-1} ; f=0,5 \mathrm{~mm} ; \alpha=6^{\circ} ; \gamma=8^{\circ}$; $r_{\varepsilon}=0,5 \mathrm{~mm}$.

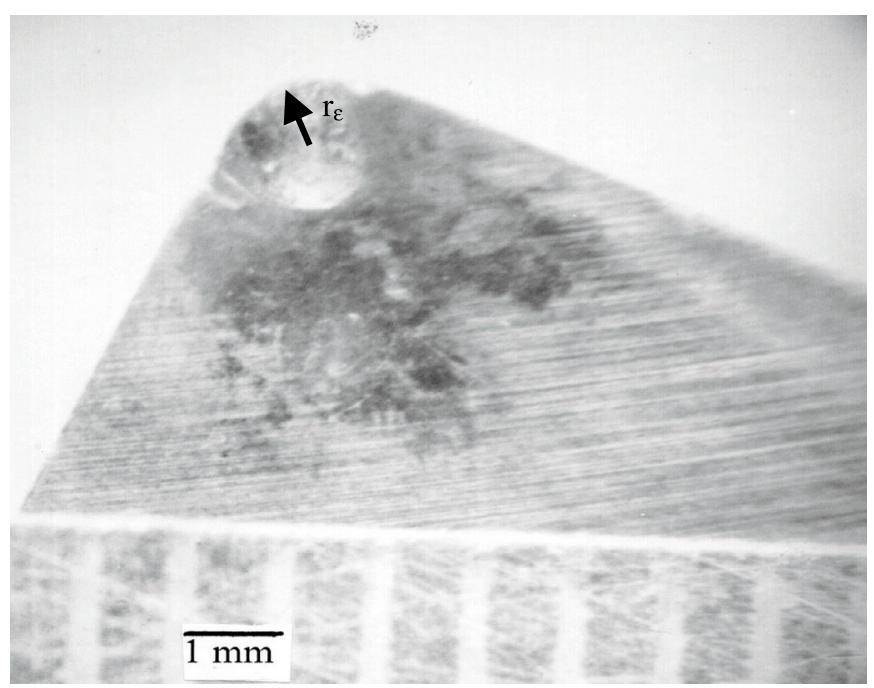

Fig. 6. Observation sur microscope optique de l'arrondi du bec de l'outil.

de Métrologie de l'ENSET d'Oran. Les résultats sont présentés sur le graphique de la figure 7 . Nous remarquons sur cette figure que la rugosité reste stable pendant la première période de $10 \mathrm{~min}$. Cette stabilité relative est due probablement à la formation préalable de l'arrondi du bec de l'outil (Fig. 6). L'apparition de l'usure en face de dépouille sous forme de creux entraîne par la suite une légère dégradation de la rugosité.

\subsubsection{Traitement mathématique des résultats}

La méthode élaborée aux sections 2 et 3 nous permet de traiter les résultats expérimentaux obtenus par les essais et représentés sur la figure 7. Selon cette figure, l'évolution de la rugosité en fonction du temps d'usinage peut être décomposée en deux périodes distinctes :

$1^{\text {re }}$ période : L'évolution de la rugosité en fonction du temps de coupe est considérée comme constante pour

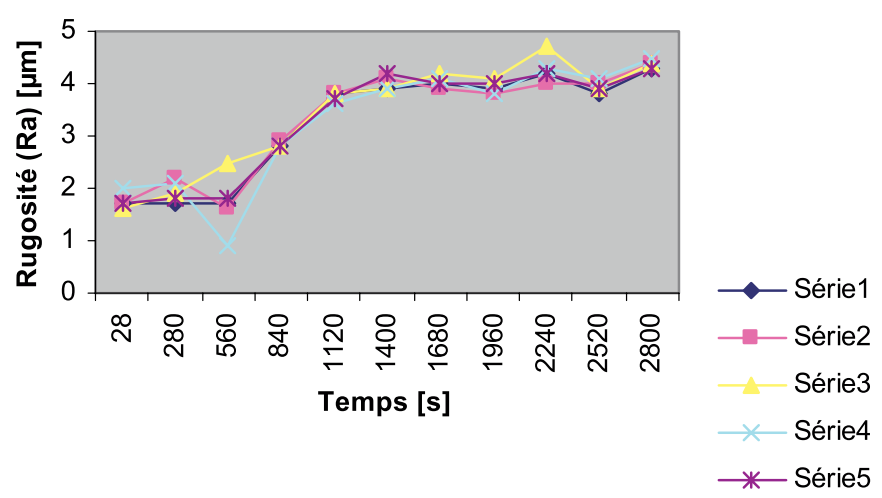

Fig. 7. Évolution de la rugosité en fonction du temps de coupe. Outil P20; $V_{\mathrm{c}}=251 \mathrm{~m} \cdot \mathrm{min}^{-1} ; s=0,1 \mathrm{~mm} \cdot \mathrm{tr}^{-1}$; $f=0,5 \mathrm{~mm} ; \alpha=6^{\circ} ; \gamma=8^{\circ} ; r_{\varepsilon}=0,5 \mathrm{~mm}$. Série 1 à 5 : essais réalisés dans les mêmes conditions.

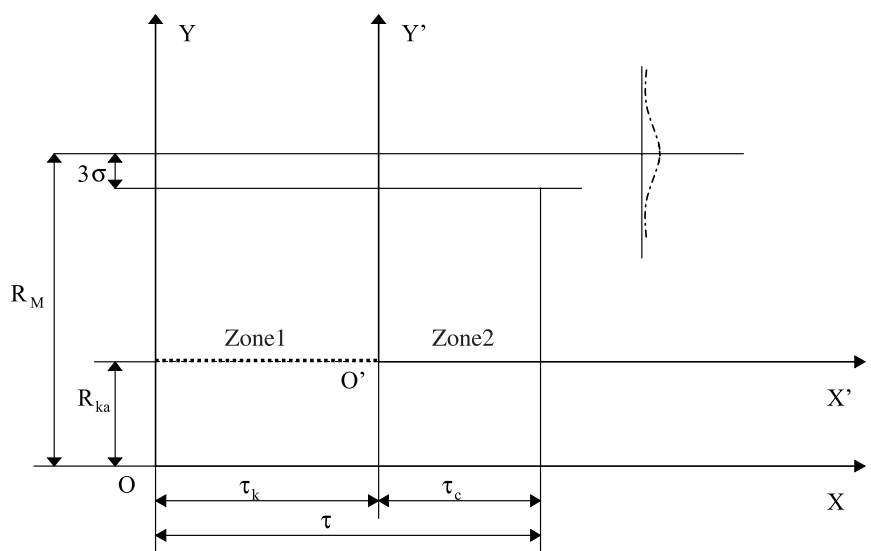

Fig. 8. Schématisation du changement de base suivant le modèle de rugosité obtenu.

le premier intervalle de temps d'usinage $(0<\tau \leq$ $560 \mathrm{~s})$.

$2^{\text {e }}$ période : La rugosité dans ce dernier intervalle (Fig. 7) varie selon la relation (9) pour un temps ( $\tau>560 \mathrm{~s})$. Étant donné que l'évolution prédite par la relation proposée décrit bien l'évolution constatée dans la deuxième période, on peut considérer que la rugosité dans la première période est constante, et faire un changement de base $\left(X \mathrm{O} Y\right.$ à $\left.X^{\prime} \mathrm{O}^{\prime} Y^{\prime}\right)$ pour la courbe de variation de la rugosité dans la deuxième période (Voir Fig. 8).

Les relations (10) et (11) suivantes nous permettent de déduire le temps du cycle d'usinage $(\tau)$, à partir d'une rugosité $R_{\mathrm{M}}$ imposée par le dessin de définition, en remplaçant les paramètres $\tau_{k}, \sigma_{R_{\mathrm{a}}}$ et $R_{k_{\mathrm{a}}}$ par leurs valeurs respectives, déterminées expérimentalement à partir de l'étude expérimentale de l'évolution de la rugosité :

$$
\begin{aligned}
& \tau=\left(\tau_{c}+\tau_{k}\right) \frac{1}{60}[\mathrm{~min}] \\
& \tau_{c}^{0,1017}=R_{\mathrm{M}}-3 \sigma_{R_{\mathrm{a}}}-R_{k_{\mathrm{a}}}
\end{aligned}
$$

La relation (11) exprime l'évolution de la rugosité dans la deuxième période, au-delà de la période initiale 
d'usinage de $560 \mathrm{~s}$; dans cette seconde période, le temps d'usinage $\left(\tau_{\mathrm{c}}\right)$ évolue suivant une courbe exponentielle dont l'exposant est 0,1017.

$\tau_{k}=560 \mathrm{~s}$ est une valeur constante, déduite à partir des résultats des essais portés sur la figure 7 ;

$\sigma_{R_{\mathrm{a}}}=0,27$ est la valeur maximale de l'écart type des rugosités, calculée à partir des résultats expérimentaux.

$R_{k_{\mathrm{a}}}=1,79$ est la valeur moyenne de la rugosité dans l'intervalle de temps $(0<\tau \leq 560 \mathrm{~s})$.

\section{Exemple}

Si la fonction d'une pièce mécanique, dont on veut prévoir l'usinage, exige une rugosité particulière, par exemple $R_{\mathrm{M}}=4,8 \mu \mathrm{m}$, on peut déduire le temps de cycle d'usinage à partir des relations (10) et (11). Nous pouvons déduire ainsi un temps $\tau$ de 48,13 min, comme temps de coupe de l'outil ; ce temps est évidemment valable dans les conditions de coupe fixées lors des essais de caractérisation : $V_{\mathrm{c}}, s, f, \alpha, \gamma, r_{\varepsilon}$.

\section{Conclusion}

Notre travail permet d'assurer facilement une rugosité inférieure à une valeur donnée par le réglage du cycle d'usinage et du temps maximum de coupe pour l'outil carbure P20 ; ce qui peut permettre d'éliminer ou de réduire le contrôle continu ou fréquentiel des pièces lors de l'usinage, d'où une diminution du prix de revient des pièces usinées.

À partir de l'examen de l'influence de certains paramètres sur la rugosité tels que les paramètres de coupe $\left(V_{\mathrm{c}}, s, f\right)$ étudiés dans le présent travail d'une part, et les paramètres géométriques définissant la forme de l'outil ( $\alpha$, $\gamma, r_{\varepsilon}$ ) fixés selon d'autres travaux, on a dégagé les valeurs permettant d'optimiser le cycle d'usinage et réalisé des essais aboutissant à une corrélation entre la rugosité et la durée de vie de l'outil exprimée par le temps d'usinage.

La rugosité obtenue en fonction du temps de coupe de l'outil évolue de deux manières distinctes. Une première période dans la quelle la rugosité est constante et une deuxième période dans la quelle la rugosité suit l'allure du modèle proposé. La première période $(\tau<560 \mathrm{~s})$, surestime les valeurs de rugosité, considérées comme constantes et connues. Pour la deuxième période, il est proposé un modèle représentant la corrélation entre la rugosité et le temps d'usinage, sous les conditions de coupe fixées lors des essais. À partir de ce modèle il est possible de limiter la durée d'utilisation de l'outil en question pour le respect d'une rugosité imposée par le constructeur et d'éviter les étapes intermédiaires pour la vérification de la rugosité lors d'un usinage conventionnel de chariotage, ce qui fait l'originalité de cette étude. Ces conditions ont été appliquées au niveau du Complexe de Machine Agricole de Sidi Bel-Abbes (Algérie) et présentées dans le cadre de travaux de recherche $[17,18]$.

\section{Références}

[1] ISO 12085, Spécification géométriques des produits (GPS). États de surfaces : Méthodes du profil. Paramètres liés aux motifs, août 1996

[2] M. Viaud, État de surface mesure de rugosité, $3^{\mathrm{e}}$ édition, CETIM Formation, 1998

[3] A. Benamar, Recherche théorique et expérimentale sur la réalisation d'une rugosité, Thèse de Magister, USTO Oran, Juin 1985

[4] St. Enache, La qualité des surface usinées, Dunod, Paris, 1972

[5] R. Levêque, Usinabilité et mécanismes endommagements des outils de coupe, Revue Matériaux et Techniques 5-6 (1999)

[6] A.M. Alam et al., Effect of machining procedures on the surface state of a low carbon steel, Scripta Materialia 37(10) (1997)

[7] C. Neagu, M. Gheorghe, Modèle mathématique pour l'étude de la rugosité imposée aux surfaces usinées, Tribotechnica, Plolesti, 1982

[8] Z. Duca, Bazele, Teoretice de preluisorilov pe mosiniunelte, Bucarest, E D P, 1969

[9] M. Bouchetara et al., Étude du phénomène d'usure des pièces des pompes d'injection par la méthode de Taguchi, Mécanique et Industries 4(3) (2003)

[10] A. Karoui, Paramétrage automatique de la gamme d'usinage, Magister en Génie Mécanique, USTO Oran, juin 2002

[11] S. Belhadi et al., Influence des conditions de coupe sur la rugosité de l'acier 45NiCrMo16 traité usiné avec un outil CBN, Séminaire International en Génie Mécanique, ENSET Oran, avril 2002

[12] P. Padilla, A. Thly, Guide des fabrications mécaniques, Dunod, Paris, 1981

[13] G. Branger, Guide du bureau des méthodes, Desforges, Paris, 1981

[14] J. Girar, Les états de surfaces en tournages, rapport du CETIM N ${ }^{\circ}$ 61, Senlis, France, 1979

[15] R. Butin, M. Pinot, Fabrication Mécaniques Technologie, Foucher, Paris, 1981

[16] C. Barlier, L. Girardin, Productique matériaux et usinage, André, Casteilla, Paris, 1986

[17] A. Benamar, G. Inglebert, Rugosité imposée pour les pièces mécaniques, Journées de Mécanique, École Militaire Polytechnique Algérie, 25-26 novembre 2000

[18] A. Benamar et al., Optimisation du procédé d'usinage à rugosité contrôlée, $3^{\text {e }}$ Journées d'Études Techniques, AMFM, Marakech, avril 2003 